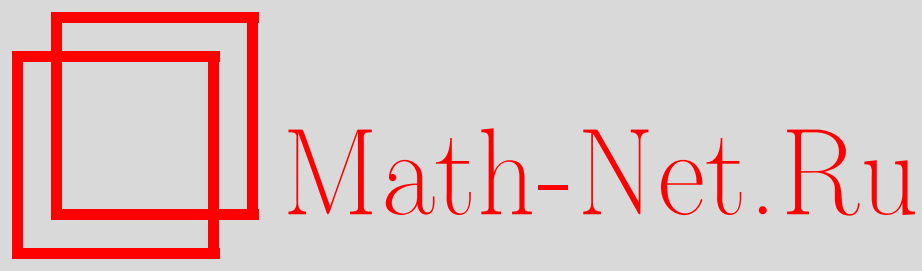

С. Д. Глызин, А. Ю. Колесов, Н. Х. Розов, Периодические решения типа бегущих волн в кольцевых цепочках однонаправленно связанных уравнений, ТМФ, 2013, том 175, номер 1, 62-83

DOI: https://doi.org/10.4213/tmf8441

Использование Общероссийского математического портала Math-Net.Ru подразумевает, что вы прочитали и согласны с пользовательским соглашением http://www.mathnet.ru/rus/agreement

Параметры загрузки:

IP : 52.205 .19 .152

26 апреля 2023 г., 16:11:03

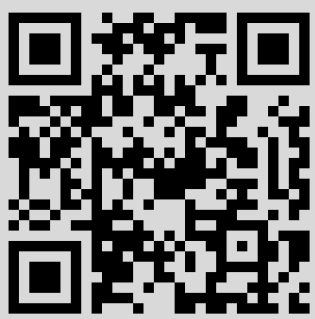




\title{
ПЕРИОДИЧЕСКИЕ РЕШЕНИЯ ТИПА БЕГУЩИХ ВОЛН В КОЛЬЦЕВЫХ ЦЕПОЧКАХ
}

\section{ОДНОНАПРАВЛЕННО СВЯЗАННЫХ УРАВНЕНИЙ}

\begin{abstract}
Рассматриваются специальные системы обыкновенных дифференциальных уравнений - так называемые кольцевые однонаправленные цепочки. Для данного класса систем разработан новый метод исследования проблемы существования и устойчивости периодических решений. Характерная особенность предлагаемого подхода заключается в том, что как при отыскании циклов, так и при анализе их свойств устойчивости используются некоторые вспомогательные системы с запаздыванием. Содержательность предложенного метода иллюстрируется на конкретном примере кольцевой нейронной сети Хопфилда.
\end{abstract}

Ключевые слова: цепочка однонаправленно связанных уравнений, система с запаздыванием, нейронная сеть Хопфилда, периодическое решение, асимптотика, устойчивость.

DOI: $10.4213 / \operatorname{tmf} 8441$

\section{1. ОБЩАЯ СХЕМА ИССЛЕДОВАНИЯ}

Кольцевой цепочкой однонаправленно связанных уравнений назовем систему вида

$$
\dot{x}_{j}=f\left(x_{j}, x_{j-1}\right), \quad j=1, \ldots, m, \quad x_{0}=x_{m},
$$

где $m \geqslant 2, x_{j}=x_{j}(t) \in \mathbb{R}^{n}$, а вектор-функция $f(x, y)$ со значениями в $\mathbb{R}^{n}$ бесконечно дифференцируема по $(x, y) \in \mathbb{R}^{n} \times \mathbb{R}^{n}$. Такого рода системы описывают функционирование различных автогенераторов (см. работы [1], [2], где рассмотрены соответствующие примеры), а также возникают при математическом моделировании в нейродинамике. В частности, к указанному типу уравнений относится известная модель кольцевой нейронной сети Хопфилда, которая подробно исследуется в настоящей статье.

*Ярославский государственный университет им. П.Г. Демидова, Ярославль, Россия. E-mail: glyzin@uniyar.ac.ru, kolesov@uniyar.ac.ru

${ }^{\dagger}$ Московский государственный университет им. М.В. Ломоносова, Москва, Россия. E-mail: fpo.mgu@mail.ru 
В дальнейшем мы будем интересоваться проблемами существования и устойчивости специальных периодических решений системы (1.1) - так называемых бегущих волн. Точнее говоря, речь пойдет о периодических решениях, допускающих представление

$$
x_{j}=x(t+(j-1) \Delta), \quad j=1, \ldots, m,
$$

где $\Delta>0$ - некоторый фазовый сдвиг.

Прежде чем строго формулировать результаты, остановимся на неформальном описании используемых нами методов. В связи с этим обратим внимание, что фигурирующая в (1.2) функция $x=x(t) \in \mathbb{R}^{n}$ с необходимостью должна удовлетворять вспомогательному уравнению с запаздыванием

$$
\dot{x}=f(x, x(t-\Delta))
$$

и иметь период $T=m \Delta / k$, где $k$ - некоторое натуральное число. И обратно, если указанное уравнение допускает требуемое периодическое решение $x(t)$, то в исходной системе (1.1) такому решению соответствует бегущая волна (1.2).

Вопрос об устойчивости цикла (1.2) некоторым образом связан с линейной системой

$$
\begin{gathered}
\dot{h}=A(t) h+\varkappa B(t) h(t-\Delta), \\
A(t)=f_{x}^{\prime}(x(t), x(t-\Delta)), \quad B(t)=f_{y}^{\prime}(x(t), x(t-\Delta)),
\end{gathered}
$$

получающейся из (1.3) при линеаризации на цикле $x(t)$ и введении дополнительного комплексного параметра $\varkappa$. Точнее говоря, для отыскания мультипликаторов бегущей волны (1.2) мы будем использовать так называемый метод подстройки по параметру $\varkappa$, в определенном смысле аналогичный процессу настройки радиоприемника на нужную станцию. Суть этого метода заключается в следующем.

Выберем из счетного семейства мультипликаторов системы (1.4) любой мультипликатор $\nu(\varkappa)$ и рассмотрим отвечающее ему уравнение

$$
\nu^{k}(\varkappa)=\varkappa^{m} .
$$

Как будет показано ниже, каждому корню $\varkappa=\varkappa_{0} \neq 0$ уравнения (1.5) соответствует мультипликатор цикла (1.2), задающийся равенством $\nu=\nu\left(\varkappa_{0}\right)$. Верно и обратное утверждение: на любой мультипликатор $\nu$ бегущей волны (1.2) можно "настроиться" за счет выбора параметра $\varkappa$. Иными словами, любой мультипликатор $\nu$ интересующего нас цикла допускает представление $\nu=\nu\left(\varkappa_{0}\right)$, где $\nu(\varkappa)$ - один из мультипликаторов уравнения (1.4), а $\varkappa_{0}$ - корень соответствующего уравнения (1.5). Таким образом, набор всех ненулевых корней уравнений (1.5) (заметим, что количество таких корней обязательно конечно) можно рассматривать как совокупность "радиочастот", на которых работают интересующие нас станции. Сам же факт "настройки на станцию" соответствует конкретному выбору одного из этих корней и получению на выходе соответствующего мультипликатора $\nu=\nu\left(\varkappa_{0}\right)$ бегущей волны $(1.2)$.

Перейдем теперь к строгому обоснованию описанных методов. Обратимся сначала к вспомогательному уравнению (1.3) и будем считать, что на некотором интервале $\left(\Delta_{1}, \Delta_{2}\right) \subset(0,+\infty)$ изменения параметра $\Delta$ оно допускает периодическое решение $x=x_{*}(t, \Delta)$ с периодом $T_{*}=T_{*}(\Delta)>0$. В этом случае справедливо следующее утверждение. 
ЛЕмма 1.1. Предположим, что найдется натуралъное $k$, при котором уравнение

$$
T_{*}(\Delta)=\frac{m \Delta}{k}
$$

имеет корень $\Delta=\Delta_{(k)} \in\left(\Delta_{1}, \Delta_{2}\right)$. Тогда в исходной системе (1.1) данному корню соответствует иикл (бегущая волна)

$$
C_{k}: x_{j}=x_{(k)}\left(t+(j-1) \Delta_{(k)}\right), \quad j=1, \ldots, m,
$$

c периодом $T_{(k)}=m \Delta_{(k)} / k$, где $x_{(k)}(t)=\left.x_{*}(t, \Delta)\right|_{\Delta=\Delta_{(k)}}$.

Для ДокАЗАТЕЛЬСТВА заметим, что, поскольку все функции

$$
x_{j}(t)=x_{(k)}\left(t+(j-1) \Delta_{(k)}\right), \quad j=1, \ldots, m,
$$

являются решениями одного и того же уравнения (1.3) при $\Delta=\Delta_{(k)}$, выполняются равенства

$$
\dot{x}_{j}(t)=f\left(x_{j}(t), x_{j}\left(t-\Delta_{(k)}\right)\right), \quad j=1, \ldots, m .
$$

Далее, учтем в (1.9) вытекающие из (1.8) соотношения

$$
\begin{aligned}
x_{j}\left(t-\Delta_{(k)}\right) & =x_{j-1}(t), \quad j=2, \ldots, m, \\
x_{1}\left(t-\Delta_{(k)}\right) & =x_{(k)}\left(t-\Delta_{(k)}\right)=x_{(k)}\left(t-\Delta_{(k)}+k T_{(k)}\right)= \\
& =x_{(k)}\left(t+(m-1) \Delta_{(k)}\right)=x_{m}(t) .
\end{aligned}
$$

В результате убеждаемся, что функции (1.8) удовлетворяют исходной системе (1.1). Лемма 1.1 доказана.

Вопрос об устойчивости цикла (1.7) сводится, очевидно, к вопросу о расположении мультипликаторов линейной системы

$$
\dot{h}_{j}=A_{(k)}\left(t+(j-1) \Delta_{(k)}\right) h_{j}+B_{(k)}\left(t+(j-1) \Delta_{(k)}\right) h_{j-1}, \quad j=1, \ldots, m,
$$

где $h_{j}=h_{j}(t) \in \mathbb{R}^{n}, h_{0}=h_{m}$, а матрицы $A_{(k)}(t), B_{(k)}(t)$ задаются равенствами

$$
A_{(k)}(t)=f_{x}^{\prime}\left(x_{(k)}(t), x_{(k)}\left(t-\Delta_{(k)}\right)\right), \quad B_{(k)}(t)=f_{y}^{\prime}\left(x_{(k)}(t), x_{(k)}\left(t-\Delta_{(k)}\right)\right) .
$$

Наряду с (1.10) в дальнейшем нам понадобится аналогичное (1.4) вспомогательное линейное уравнение

$$
\dot{h}=A_{(k)}(t) h+\varkappa B_{(k)}(t) h\left(t-\Delta_{(k)}\right),
$$

где $h(t) \in \mathbb{C}^{n}, \varkappa-$ произвольный комплексный параметр. Точнее говоря, нас будут интересовать его мультипликаторы $\nu_{s}(\varkappa), s=1,2, \ldots$, пронумерованные в порядке убывания модулей.

Поясним смысл термина "мультипликатор" применительно к уравнению с запаздыванием (1.12). В связи с этим рассмотрим пространство $E=C\left(\left[-\Delta_{(k)}, 0\right] ; \mathbb{C}^{n}\right)$ непрерывных при $-\Delta_{(k)} \leqslant t \leqslant 0$ вектор-функций $h^{0}(t)=\left(h_{1}^{0}(t), \ldots, h_{n}^{0}(t)\right)^{\mathrm{T}}$ с нормой

$$
\left\|h^{0}\right\|_{E}=\max _{1 \leqslant l \leqslant n} \max _{-\Delta_{(k)} \leqslant t \leqslant 0}\left|h_{l}^{0}(t)\right| .
$$


Оператором монодромии уравнения (1.12) назовем линейный ограниченный оператор $V: E \rightarrow E$, действующий на произвольную функцию $h^{0}(t) \in E$ по правилу

$$
V h^{0}=h\left(t+m \Delta_{(k)} / k\right), \quad-\Delta_{(k)} \leqslant t \leqslant 0,
$$

где $h(t)$ - решение уравнения (1.12) на отрезке времени $0 \leqslant t \leqslant m \Delta_{(k)} / k$ с начальной функцией $h^{0}(t),-\Delta_{(k)} \leqslant t \leqslant 0$. Отметим, что спектр этого оператора заведомо дискретен, так как некоторая его степень компактна (в случае $m / k \geqslant 1$ компактен и сам $V$ ). Что касается мультипликаторов уравнения (1.12), то таковыми по аналогии со случаем обыкновенных дифференциальных уравнений будем называть собственные значения оператора (1.13).

Остановимся на вопросе о связи между мультипликаторами систем (1.10) и (1.12). Имеет место следующая

ЛЕмма 1.2. Каждъй мулътипликатор д системъ (1.10) допускает представление

$$
\nu=\varkappa^{m / k}
$$

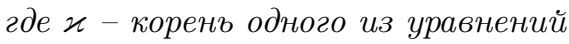

$$
\nu_{s}^{k}(\varkappa)=\varkappa^{m}, \quad s \in \mathbb{N} .
$$

И обратно, если при некотором $s=s_{0}$ уравнение (1.15) имеет корень $\varkappa=\varkappa_{0} \neq 0$, то у исходной системъ (1.10) существует мультипликатор $\nu=\nu_{s_{0}}\left(\varkappa_{0}\right)$.

ДокАЗАТЕльство. Зафиксируем произвольный мультипликатор $\nu=\rho e^{i \varphi}, \rho>0$, $0 \leqslant \varphi<2 \pi$, системы (1.10) и предположим, что он является простым. В этом случае ему отвечает единственное (с точностью до множителя) решение Ляпунова-Флоке вида

$$
h_{j}=e^{\alpha t} h_{*, j}(t), \quad h_{*, j}(t) \in \mathbb{C}^{n}, \quad h_{*, j}\left(t+m \Delta_{(k)} / k\right) \equiv h_{j, *}(t), \quad j=1, \ldots, m,
$$

где

$$
\alpha=\frac{k}{m \Delta_{(k)}}(\ln \rho+i \varphi) .
$$

Отметим далее, что, поскольку система (1.10) инвариантна относительно замен

$$
t-\Delta_{(k)} \rightarrow t, \quad h_{j-1} \rightarrow h_{j}, \quad j=1, \ldots, m,
$$

под действием этих замен решение (1.16) (в силу его единственности) перейдет в решение

$$
h_{j}=\lambda e^{\alpha\left(t+\Delta_{(k)}\right)} h_{*, j}(t), \quad j=1, \ldots, m,
$$

где $\lambda \neq 0$ - некоторая комплексная постоянная. Таким образом, имеет место равенство

$$
\Lambda h_{*}\left(t+\Delta_{(k)}\right)=\lambda h_{*}(t),
$$

3 Теоретическая и математическая физика, т. 175, № 1, 2013 г. 
где $h_{*}(t)=\left(h_{*, 1}(t), \ldots, h_{*, m}(t)\right)^{\mathrm{T}}$, а элементами квадратной $(n m)$-мерной матрицы

$$
\Lambda=\left(\begin{array}{ccccc}
0 & 0 & \cdots & 0 & I \\
I & 0 & \cdots & 0 & 0 \\
0 & I & \ddots & \vdots & 0 \\
\vdots & \ddots & \ddots & 0 & \vdots \\
0 & \cdots & 0 & I & 0
\end{array}\right)
$$

являются нулевые и единичные матрицы размера $n \times n$.

Из установленного выше соотношения (1.18) следует, что

$$
h_{*, m-j}(t)=\lambda^{j+1} h_{*, 1}\left(t-(j+1) \Delta_{(k)}\right), \quad j=0,1, \ldots, m-2, \quad \lambda^{m}=1 .
$$

Компонента $h_{*, 1}(t)$ в силу (1.10) является решением линейного неоднородного уравнения

$$
\dot{h}=-\alpha h+A_{(k)}(t) h+B_{(k)}(t) h_{*, m}(t) .
$$

При этом функция $h_{*, m}(t)$, в свою очередь, выражается через $h_{*, 1}(t)$ посредством равенства $h_{*, m}(t)=\lambda h_{*, 1}\left(t-\Delta_{(k)}\right)\left(\right.$ см. (1.19)). Поэтому компонента $h_{*, 1}(t)$ удовлетворяет также уравнению с запаздыванием

$$
\dot{h}=-\alpha h+A_{(k)}(t) h+\lambda B_{(k)}(t) h\left(t-\Delta_{(k)}\right) .
$$

Проделанные построения показывают, что уравнение (1.20) заведомо имеет единичный мультипликатор. Далее сделаем в этом уравнении замену $e^{\alpha t} h \rightarrow h$. В peзультате единичный мультипликатор перейдет в $e^{m \alpha \Delta_{(k)} / k}$, а само уравнение $(1.20)-$ в уравнение $(1.12)$ при $\varkappa=\lambda e^{\alpha \Delta_{(k)}}$. Таким образом, с необходимостью найдется номер $s$, для которого

$$
\left.\nu_{s}(\varkappa)\right|_{\varkappa=\lambda e^{\alpha \Delta_{(k)}}}=e^{m \alpha \Delta_{(k)} / k}=\nu .
$$

Отсюда и из очевидного равенства $\nu^{k}=\varkappa^{m}$ вытекают соотношения (1.14), (1.15).

В случае, когда мультипликатор $\nu$ кратный, рассуждения аналогичны. Действительно, пусть данному мультипликатору отвечают ровно $p$ линейно независимых решений Ляпунова-Флоке. Тогда эти решения можно записать в матричной форме $e^{\alpha t} H(t)$, где столбцами матрицы $H(t)$ размера $m n \times p$ являются линейно независимые $T_{(k)}$-периодические вектор-функции. Далее, в силу инвариантности системы (1.10) под действием замен (1.17) здесь вместо (1.18) будет выполняться равенство

$$
\Lambda H\left(t+\Delta_{(k)}\right)=H(t) D
$$

с некоторой невырожденной постоянной матрицей $D$ размера $p \times p$.

Свойство (1.21) позволяет свести проблему обоснования формул (1.14), (1.15) к предыдущему случаю. Для того чтобы сделать это, фиксируем некоторое собственное значение $\lambda$ матрицы $D$, а через $е$ обозначим отвечающий ему собственный вектор. Тогда, как нетрудно видеть, для вектор-функции $h_{*}(t)=H(t) e$ справедливо соотношение (1.18). Дальнейшие рассуждения совпадают с изложенными выше. 
Итак, мы установили, что любой мультипликатор $\nu$ системы (1.10) может быть представлен в виде (1.14), где $\varkappa$ удовлетворяет одному из уравнений (1.15). Убедимся теперь в справедливости обратного утверждения. В связи с этим предположим, что уравнение (1.15) с номером $s=s_{0}$ допускает корень $\varkappa=\varkappa_{0} \neq 0$. Тогда уравнение

$$
\dot{h}=-\alpha h+A_{(k)}(t) h+\varkappa_{0} e^{-\alpha \Delta_{(k)}} B_{(k)}(t) h\left(t-\Delta_{(k)}\right)
$$

при

$$
\alpha=\frac{k}{m \Delta_{(k)}}\left(\ln \rho_{0}+i \varphi_{0}\right), \quad \rho_{0}>0, \quad 0 \leqslant \varphi_{0}<2 \pi,
$$

где $\rho_{0} e^{i \varphi_{0}}=\nu_{s_{0}}\left(\varkappa_{0}\right)$, имеет нетривиальное $T_{(k)}$-периодическое решение $\tilde{h}(t)$. Введем в рассмотрение величину

$$
\lambda=\varkappa_{0} e^{-\alpha \Delta(k)}
$$

и заметим, что в силу (1.23) и соотношения $\varkappa_{0}^{m}=\nu_{s_{0}}^{k}\left(\varkappa_{0}\right)$ указанное значение параметра $\lambda$ удовлетворяет требуемому равенству $\lambda^{m}=1$ (см. (1.19)). А отсюда, в свою очередь, следует, что при выбранном $\lambda$ уравнения (1.22) и (1.20) совпадают.

На заключительном этапе доказательства введем функцию $h_{*, 1}(t)=\tilde{h}(t)$, а остальные компоненты $h_{*, j}(t), j=2, \ldots, m$, определим посредством равенств (1.19), (1.24). Из установленной выше связи между уравнениями (1.22) и (1.20) вытекает, что в итоге получится решение Ляпунова-Флоке вида (1.16) исходной системы (1.10), отвечающее мультипликатору

$$
\nu=e^{m \alpha \Delta_{(k)} / k}=\nu_{s_{0}}\left(\varkappa_{0}\right) .
$$

Лемма 1.2 доказана.

Доказанные леммы позволяют сформулировать некую общую методику исследования периодических решений типа бегущих волн в кольцевых системах (1.1). Действительно, вопрос о существовании циклов вида (1.2) сводится к отысканию цикла $x_{*}(t, \Delta)$ вспомогательного уравнения с запаздыванием (1.3) и к нахождению корней уравнений (1.6). Что касается вопроса об устойчивости бегущих волн, то он решается отдельно и в силу леммы 1.2 состоит в анализе расположения корней уравнений (1.15). Следует также отметить, что, хотя количество уравнений в системе (1.15), вообще говоря, счетно, совокупность всех их ненулевых корней заведомо конечна (в противном случае конечномерная система (1.10) имела бы счетное число различных мультипликаторов, что невозможно).

Достаточно ясно, что проблемы анализа вспомогательных уравнений (1.3), (1.12), лежащих в основе описанной выше методики, в общем случае нелокальны. Но тем не менее в некоторых ситуациях, когда есть возможность применить какие-либо асимптотические методы, с указанными проблемами удается справиться. Именно такая ситуация реализуется в рассматриваемой ниже математической модели кольцевой нейронной сети Хопфилда.

Системой Хопфилда принято называть предложенную в работе [3] систему обыкновенных дифференциальных уравнений

$$
\dot{u}_{j}=-\mu_{j} u_{j}+\sum_{i=1}^{m} a_{i j} f_{i}\left(u_{i}\right)+I_{j}, \quad j=1, \ldots, m,
$$


являющуюся математической моделью простейшей нейронной сети. Здесь $u_{j}(t)-$ мембранные потенциалы нейронов, $\mu_{j}=$ const $>0-$ коэффициенты затухания за счет токов утечки, $a_{i j}=$ const $\in \mathbb{R}$ - синаптические веса, $I_{j}=$ const $\in \mathbb{R}-$ внешние токи смещения, а гладкие функции $f_{j}(u), u \in \mathbb{R}$, представляющие собой вольт-амперные характеристики нелинейных элементов, таковы, что

$$
\lim _{u \rightarrow-\infty} f_{j}(u)=0, \quad \lim _{u \rightarrow+\infty} f_{j}(u)=1 .
$$

Достаточно подробное описание свойств этой системы, а также ее вывод можно найти в монографии [4].

В дальнейшем нас будет интересовать кольцевая система

$$
\dot{u}_{j}=-\mu u_{j}+\lambda\left[1-(a+1) f\left(u_{j-1}\right)\right], \quad j=1, \ldots, m, \quad u_{0}=u_{m},
$$

являющаяся частным случаем системы (1.25). Здесь $\mu, a=$ const $>0$, а параметр $\lambda>0$ предполагается большим (с биофизической точки зрения это означает, что электрические процессы в нейронной сети происходят быстро). Что касается нелинейности функции $f(u) \in C^{\infty}(\mathbb{R})$, то в соответствии с требованиями (1.26) мы считаем выполненными асимптотические равенства

$$
f(u)=\sum_{l=1}^{\infty} \frac{c_{l}^{-}}{u^{l}}, \quad u \rightarrow-\infty, \quad f(u)=1+\sum_{l=1}^{\infty} \frac{c_{l}^{+}}{u^{l}}, \quad u \rightarrow+\infty .
$$

Предполагаем также, что представления (1.28) остаются в силе при дифференцировании по $и$ любое число раз. Типичными представителями таких функций являются

$$
f(u)=\frac{\operatorname{arctg} u+\pi / 2}{\pi}, \quad f(u)=\frac{1+u / \sqrt{u^{2}+1}}{2}, \quad f(u)=\frac{1}{1+e^{-u}}
$$

(в последнем случае все коэффициенты $c_{l}^{ \pm}, l \geqslant 1$, равны нулю).

Цель настоящей статьи заключается в том, чтобы на примере системы (1.27) наглядно проиллюстрировать эффективность предложенных нами новых методов исследования периодических решений типа бегущих волн.

\section{2. АНАЛИЗ ВСПОМОГАТЕЛЬНЫХ УРАВНЕНИЙ}

Обратимся сначала к вспомогательному уравнению (1.3), которое в случае системы (1.27) имеет вид

$$
\dot{u}=-\mu u+\lambda[1-(a+1) f(u(t-\Delta))] .
$$

Ниже будет установлено, что при любом фиксированном значении запаздывания $\Delta>0$ и при всех $\lambda \gg 1$ это уравнение имеет экспоненциально орбитально устойчивый релаксационный цикл.

Для удобства последующего анализа выполним в (2.1) замену $u=\lambda x$. В результате интересующее нас уравнение примет вид

$$
\dot{x}=-\mu x+1-(a+1) f(x(t-\Delta) / \varepsilon),
$$


где $\varepsilon=1 / \lambda \ll 1$. Далее, опираясь на свойства (1.28) функции $f(u)$, замечаем, что

$$
\lim _{\varepsilon \rightarrow 0} f(x / \varepsilon)=F(x) \stackrel{\text { def }}{=} \begin{cases}0 & \text { при } x<0, \\ 1 & \text { при } x>0 .\end{cases}
$$

Наконец, с учетом соотношения (2.3) получаем из уравнения (2.2) предельное релейное уравнение с запаздыванием

$$
\dot{x}=-\mu x+1-(a+1) F(x(t-\Delta)) .
$$

Как и в работах [5]-[7], понятие решения уравнения (2.4) определим конструктивно. В связи с этим фиксируем некоторое достаточно малое $\sigma_{0}>0$ (оценка сверху для $\sigma_{0}$ будет уточнена далее), рассмотрим множество функций

$$
\left\{\varphi(t) \in C\left[-\Delta-\sigma_{0},-\sigma_{0}\right]: \varphi(t)<0, t \in\left[-\Delta-\sigma_{0},-\sigma_{0}\right], \quad \varphi\left(-\sigma_{0}\right)=\theta\left(-\sigma_{0}\right)\right\},
$$

где

$$
\theta(t)=\frac{1-e^{-\mu t}}{\mu},
$$

и обозначим через $x_{\varphi}(t), t \geqslant-\sigma_{0}$, решение уравнения $(2.4)$ с произвольной начальной функцией $\varphi$ из множества (2.5).

Рассмотрим сначала отрезок $t \in\left[-\sigma_{0}, \Delta-\sigma_{0}\right]$ и заметим, что при указанных значениях $t$ выполняется неравенство $\varphi(t-\Delta)<0$. Поэтому на данном промежутке решение $x_{\varphi}(t)$ совпадает с решением задачи Коши

$$
\dot{x}=-\mu x+1,\left.\quad x\right|_{t=-\sigma_{0}}=\theta\left(-\sigma_{0}\right)
$$

и, следовательно,

$$
x_{\varphi}(t)=\theta(t),
$$

где $\theta(t)$ - функция (2.6). Ясно также, что формула (2.8) сохраняется до тех пор, пока $x_{\varphi}(t-\Delta)<0$. Тем самым она справедлива на полуинтервале $-\sigma_{0} \leqslant t<\Delta$.

При $t=\Delta$ первый раз происходит переключение, и при $t \geqslant \Delta$ решение $x_{\varphi}(t)$ определяется уже из задачи Коши

$$
\dot{x}=-\mu x-a,\left.\quad x\right|_{t=\Delta}=\theta(\Delta),
$$

т. е. записывается как

$$
x_{\varphi}(t)=\theta(\Delta) e^{-\mu(t-\Delta)}-a \theta(t-\Delta) .
$$

В свою очередь, соотношения (2.9), (2.10) остаются в силе, пока $x_{\varphi}(t-\Delta)>0$, т. е. до очередного момента переключения $t=t_{0}+\Delta$, где $t_{0}>\Delta-$ корень уравнения

$$
\theta(\Delta) e^{-\mu(t-\Delta)}-a \theta(t-\Delta)=0,
$$

задающийся равенством

$$
t_{0}=\Delta+\frac{1}{\mu} \ln \left(1+\frac{1}{a}\left(1-e^{(-\mu \Delta)}\right)\right) .
$$


При $t>t_{0}+\Delta$ мы имеем аналогичную (2.7) задачу Коши

$$
\dot{x}=-\mu x+1,\left.\quad x\right|_{t=t_{0}+\Delta}=\theta(\Delta) e^{-\mu t_{0}}-a \theta\left(t_{0}\right),
$$

следовательно, справедливо аналогичное (2.8) равенство

$$
x_{\varphi}(t)=\left(\theta(\Delta) e^{-\mu t_{0}}-a \theta\left(t_{0}\right)\right) e^{-\mu\left(t-t_{0}-\Delta\right)}+\theta\left(t-t_{0}-\Delta\right) .
$$

Что касается соотношений $(2.12),(2.13)$, то они сохраняют силу при априорном условии $x_{\varphi}(t-\Delta)<0$, т. е. до следующего момента переключения $t=T_{0}+\Delta$, где $t=T_{0}-$ корень уравнения

$$
\left(\theta(\Delta) e^{-\mu t_{0}}-a \theta\left(t_{0}\right)\right) e^{-\mu\left(t-t_{0}-\Delta\right)}+\theta\left(t-t_{0}-\Delta\right)=0 .
$$

Несложный подсчет показывает, что для этого корня справедлива явная формула

$$
T_{0}=t_{0}+\Delta+\frac{1}{\mu} \ln \left(1+a\left(1-e^{-\mu \Delta}\right)\right)
$$

Отметим также, что после нахождения $T_{0}$ равенство $(2.13)$ преобразуется к более компактному виду

$$
x_{\varphi}(t)=\theta\left(t-T_{0}\right) .
$$

Распорядимся теперь имеющимся в запасе свободным параметром $\sigma_{0}($ см. $(2.5))$. Из приведенных выше построений следует, что при условии $\sigma_{0}<T_{0}-t_{0}-\Delta$, которое всюду ниже считаем выполненным, функция $x_{\varphi}\left(t+T_{0}\right),-\Delta-\sigma_{0} \leqslant t \leqslant-\sigma_{0}$, принадлежит введенному ранее множеству (2.5). А это значит, что на промежутках времени

$$
l T_{0}-\sigma_{0} \leqslant t \leqslant(l+1) T_{0}-\sigma_{0}, \quad l=1,2, \ldots,
$$

весь описанный процесс нахождения $x_{\varphi}(t)$ циклически повторяется. Следовательно, при всех $t \geqslant-\sigma_{0}$ каждое решение $x_{\varphi}(t)$ с начальным условием (2.5) совпадает с одной и той же $T_{0}$-периодической функцией $x_{0}(t)$, для которой в силу $(2.8)-(2.15)$ справедливы равенства

$$
x_{0}(t)= \begin{cases}\theta(t) & \text { при } 0 \leqslant t \leqslant \Delta, \\ \theta(\Delta) e^{-\mu(t-\Delta)}-a \theta(t-\Delta) & \text { при } \Delta \leqslant t \leqslant t_{0}+\Delta, \\ \theta\left(t-T_{0}\right) & \text { при } t_{0}+\Delta \leqslant t \leqslant T_{0} .\end{cases}
$$

График этой функции при $a=2, \mu=0.8, \Delta=1$ показан на рис. 1 .

Перейдем к вопросу о связи между периодическими решениями уравнений (2.2) и (2.4). Из общих результатов статьи [5] о $C^{1}$-близости траекторий релейной и релаксационной систем вытекает следующее утверждение.

Теорема 2.1. Найдется такое достаточно малое значение $\varepsilon_{0}>0$, что при всех $0<\varepsilon \leqslant \varepsilon_{0}$ уравнение (2.2) имеет орбитально экспоненииально устойчивыи иикл $x_{*}(t, \varepsilon), x_{*}\left(-\sigma_{0}, \varepsilon\right) \equiv \theta\left(-\sigma_{0}\right)$, с периодом $T_{*}(\varepsilon)$. Для этого иикла справедливы асимптотические равенства

$$
\begin{aligned}
\max _{-\sigma_{0} \leqslant t \leqslant T_{*}(\varepsilon)-\sigma_{0}}\left|x_{*}(t, \varepsilon)-x_{0}(t)\right| & =O\left(\varepsilon \ln \frac{1}{\varepsilon}\right), \quad T_{*}(\varepsilon)=T_{0}+O\left(\varepsilon \ln \frac{1}{\varepsilon}\right), \\
\max _{t \in \Sigma}\left|\dot{x}_{*}(t, \varepsilon)-\dot{x}_{0}(t)\right| & =O(\sqrt{\varepsilon}),
\end{aligned}
$$




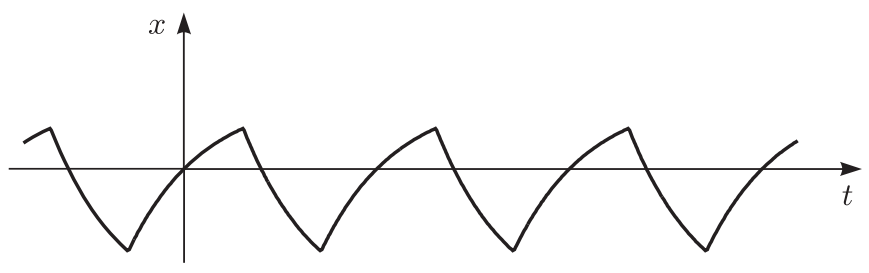

Рис. 1

где множество $\Sigma$ представляет собой отрезок $\left[-\sigma_{0}, T_{*}(\varepsilon)-\sigma_{0}\right]$ с исключенными интервалами $(\Delta-\sqrt{\varepsilon}, \Delta+\sqrt{\varepsilon}),\left(t_{0}+\Delta-\sqrt{\varepsilon}, t_{0}+\Delta+\sqrt{\varepsilon}\right)$.

Обратимся теперь к аналогичному (1.12) вспомогательному линейному уравнению

$$
\dot{h}=-\mu h+\varkappa A(t, \varepsilon) h(t-\Delta)
$$

с произвольно фиксированным запаздыванием $\Delta>0$. Здесь $0<\varepsilon \ll 1, \varkappa-$ комплексный параметр, а коэффициент $A(t, \varepsilon)$, являющийся аналогом $B_{(k)}(t)$ из $(1.11)$, задан равенством

$$
A(t, \varepsilon)=-\frac{a+1}{\varepsilon} f^{\prime}\left(x_{*}(t-\Delta, \varepsilon) / \varepsilon\right),
$$

где $x_{*}(t, \varepsilon)$ - периодическое решение уравнения $(2.2)$, доставляемое теоремой 2.1.

В связи с леммой 1.2 актуален вопрос об асимптотическом поведении при $\varepsilon \rightarrow 0$ мультипликаторов уравнения (2.18). Для его решения нам потребуются два вспомогательных утверждения. В первом из них речь идет о необходимых в дальнейшем свойствах коэффициента (2.19).

Лемма 2.1. Найдутся такое достаточно малое $\varepsilon_{0}>0$ и такие не зависящие от в постоянные $M_{1}, M_{2}>0$, что при всех $0<\varepsilon \leqslant \varepsilon_{0}$ выполняются неравенства

$$
\max _{-\sigma_{0} \leqslant t \leqslant \Delta-\sigma_{0}}|A(t, \varepsilon)| \leqslant M_{1} \varepsilon, \quad \int_{\Sigma}|A(t, \varepsilon)| d t \leqslant M_{2} \sqrt{\varepsilon},
$$

где множество $\Sigma$ задано в условии теоремы 2.1. Кроме этого, справедливы асимптотические формуль

$$
\begin{aligned}
& \int_{\Delta-\sqrt{\varepsilon}}^{\Delta+\sqrt{\varepsilon}} A(t, \varepsilon) d t=-a-1+O(\sqrt{\varepsilon}), \\
& \int_{\Delta-\sqrt{\varepsilon}}^{\Delta+\sqrt{\varepsilon}}|A(t, \varepsilon)| d t=(a+1) \int_{-\infty}^{+\infty}\left|f^{\prime}(\tau)\right| d \tau+O(\sqrt{\varepsilon}), \\
& \int_{t_{0}+\Delta-\sqrt{\varepsilon}}^{t_{0}+\Delta+\sqrt{\varepsilon}} A(t, \varepsilon) d t=-1-\frac{1}{a}+O(\sqrt{\varepsilon}), \\
& \int_{t_{0}+\Delta-\sqrt{\varepsilon}}^{t_{0}+\Delta+\sqrt{\varepsilon}}|A(t, \varepsilon)| d t=\left(1+\frac{1}{a}\right) \int_{-\infty}^{+\infty}\left|f^{\prime}(\tau)\right| d \tau+O(\sqrt{\varepsilon}),
\end{aligned}
$$

где $t_{0}$ - момент времени (2.11). 
ДоКАЗАТЕЛЬСтво. Из свойств (1.28) функции $f(u)$ последовательно выводим следующие оценки:

$$
\begin{gathered}
\left|f^{\prime}(u)\right| \leqslant \frac{M}{1+u^{2}}, \quad\left|f^{\prime \prime}(u)\right| \leqslant \frac{M}{1+|u|^{3}}, \quad u \in \mathbb{R}, \\
\left|f^{\prime}\left(u_{1}\right)-f^{\prime}\left(u_{2}\right)\right| \leqslant \frac{M}{1+\min \left(\left|u_{1}\right|^{3},\left|u_{2}\right|^{3}\right)}\left|u_{1}-u_{2}\right|, \quad u_{1}, u_{2} \in \mathbb{R},
\end{gathered}
$$

где здесь и ниже одной и той же буквой $M$ мы обозначаем различные универсальные (зависящие, быть может, только от $\Delta$ ) положительные постоянные, точные значения которых несущественны. Далее, объединяя оценку для $f^{\prime}(u)$ из $(2.23)$ с асимптотическими свойствами периодического решения $x_{*}(t, \varepsilon)$ (см. $\left.(2.17)\right)$, заключаем, что при $t \in \Sigma$

$$
|A(t, \varepsilon)| \leqslant \frac{M}{\varepsilon}\left(\frac{1}{1+(t-\Delta)^{2} / \varepsilon^{2}}+\frac{1}{1+\left(t-t_{0}-\Delta\right)^{2} / \varepsilon^{2}}\right) .
$$

Отсюда требуемые неравенства (2.20) вытекают очевидным образом.

Обратимся к асимптотическим соотношениям (2.21), (2.22) и докажем, к примеру, первые из них. С этой целью перейдем на промежутке $\Delta-\sqrt{\varepsilon} \leqslant t \leqslant \Delta+\sqrt{\varepsilon}$ к переменной $\tau$ по формуле $\tau=\left(t-\tau_{*}-\Delta\right) / \varepsilon$, где $\tau_{*}-$ корень уравнения $x_{*}(t, \varepsilon)=0$ из отрезка $-\sigma_{0} \leqslant t \leqslant \Delta-\sqrt{\varepsilon}$. Привлекая вытекающие из $(2.16),(2.17)$ формулы

$$
x_{*}(t, \varepsilon)=\theta(t)+O\left(\varepsilon \ln \frac{1}{\varepsilon}\right), \quad \dot{x}_{*}(t, \varepsilon)=\dot{\theta}(t)+O(\sqrt{\varepsilon}), \quad-\sigma_{0} \leqslant t \leqslant \Delta-\sqrt{\varepsilon},
$$

убеждаемся, что корень $\tau_{*}$ определяется однозначно и допускает асимптотическое представление

$$
\tau_{*}=O\left(\varepsilon \ln \frac{1}{\varepsilon}\right)
$$

Переменная $\tau$ меняется на отрезке $\left[\gamma_{-}(\varepsilon), \gamma_{+}(\varepsilon)\right]$, где в силу равенства $(2.25)$

$$
\gamma_{ \pm}(\varepsilon)= \pm \frac{1}{\sqrt{\varepsilon}}-\frac{\tau_{*}}{\varepsilon}= \pm \frac{1}{\sqrt{\varepsilon}}+O\left(\ln \frac{1}{\varepsilon}\right)
$$

После перехода к новому времени $\tau$ для функции

$$
\frac{x_{*}(t-\Delta, \varepsilon)}{\varepsilon}=\frac{x_{*}\left(\tau_{*}+\varepsilon \tau, \varepsilon\right)}{\varepsilon}
$$

с учетом равенства $x_{*}\left(\tau_{*}, \varepsilon\right)=0$ получаем представление

$$
\frac{x_{*}\left(\tau_{*}+\varepsilon \tau, \varepsilon\right)}{\varepsilon}=\dot{x}_{*}\left(\tau_{*}+\varepsilon \bar{\tau}, \varepsilon\right) \tau,
$$

где значение $\bar{\tau}$ таково, что $|\bar{\tau}| \leqslant|\tau|$. Учитывая далее в $(2.27)$ асимптотические формулы (2.24)-(2.26) и очевидную оценку

$$
|\bar{\tau}| \leqslant \max \left(\left|\gamma_{-}(\varepsilon)\right|,\left|\gamma_{+}(\varepsilon)\right|\right) \leqslant \frac{1}{\sqrt{\varepsilon}}+\frac{\left|\tau_{*}\right|}{\varepsilon}=\frac{1}{\sqrt{\varepsilon}}+O\left(\ln \frac{1}{\varepsilon}\right),
$$


приходим к выводу, что

$$
\frac{x_{*}\left(\tau_{*}+\varepsilon \tau, \varepsilon\right)}{\varepsilon}=(1+O(\sqrt{\varepsilon})) \tau,
$$

где оценка остатка равномерна по $\tau \in\left[\gamma_{-}(\varepsilon), \gamma_{+}(\varepsilon)\right]$.

На заключительном этапе воспользуемся третьим неравенством в $(2.23)$, из которого в силу (2.28) вытекает, что

$$
\left|f^{\prime}\left(x_{*}\left(\tau_{*}+\varepsilon \tau, \varepsilon\right) / \varepsilon\right)-f^{\prime}(\tau)\right| \leqslant \frac{M|\tau| \sqrt{\varepsilon}}{1+|\tau|^{3}}, \quad \tau \in\left[\gamma_{-}(\varepsilon), \gamma_{+}(\varepsilon)\right] .
$$

Привлечем затем оценку (2.29) непосредственно к вычислению интегралов из (2.21). В результате убеждаемся, что

$$
\begin{aligned}
\int_{\Delta-\sqrt{\varepsilon}}^{\Delta+\sqrt{\varepsilon}} A(t, \varepsilon) d t & =-(a+1) \int_{\gamma_{-}(\varepsilon)}^{\gamma_{+}(\varepsilon)} f^{\prime}(\tau) d \tau+O(\sqrt{\varepsilon})=-a-1+O(\sqrt{\varepsilon}), \\
\int_{\Delta-\sqrt{\varepsilon}}^{\Delta+\sqrt{\varepsilon}}|A(t, \varepsilon)| d t & =(a+1) \int_{\gamma_{-}(\varepsilon)}^{\gamma_{+}(\varepsilon)}\left|f^{\prime}(\tau)\right| d \tau+O(\sqrt{\varepsilon})= \\
& =(a+1) \int_{-\infty}^{+\infty}\left|f^{\prime}(\tau)\right| d \tau+O(\sqrt{\varepsilon}) .
\end{aligned}
$$

Лемма 2.1 доказана.

Перед формулировкой следующего утверждения введем в рассмотрение банахово пространство $C_{0}$ непрерывных на отрезке $-\Delta-\sigma_{0} \leqslant t \leqslant-\sigma_{0}$ комплекснозначных функций $h_{0}(t), h_{0}\left(-\sigma_{0}\right)=0$, с нормой $\left\|h_{0}\right\|=\max _{-\Delta-\sigma_{0} \leqslant t \leqslant-\sigma_{0}}\left|h_{0}(t)\right|$. Обозначим через $h(t, \varepsilon), t \geqslant-\sigma_{0}$, решение уравнения (2.18) с произвольным начальным условием $h_{0}(t),-\Delta-\sigma_{0} \leqslant t \leqslant-\sigma_{0}$, из пространства $C_{0}$.

ЛЕмма 2.2. Для каждого значения $r>0$ можно указать такие постоянные $M=M(r)>0, \varepsilon_{0}=\varepsilon_{0}(r)>0$, что для любого $0<\varepsilon \leqslant \varepsilon_{0}$ при всех значениях $\varkappa \in B(r) \stackrel{\text { def }}{=}\{\varkappa \in \mathbb{C}:|\varkappa| \leqslant r\}$ и при любъх $h_{0} \in C_{0}$ имеет место оценка

$$
\max _{-\sigma_{0} \leqslant t \leqslant T_{*}(\varepsilon)-\sigma_{0}}|h(t, \varepsilon)| \leqslant M \varepsilon\left\|h_{0}\right\| .
$$

ДокАЗАТЕЛЬство. Фиксируем произвольно положительное $r$ и будем считать, что $\varkappa \in B(r)$. Рассмотрим отрезок $-\sigma_{0} \leqslant t \leqslant \Delta-\sigma_{0}$, на котором для коэффициента $A(t, \varepsilon)$ справедлива первая оценка (2.20). Учитывая эту оценку в явной формуле

$$
h(t, \varepsilon)=\varkappa \int_{-\sigma_{0}}^{t} e^{-\mu(t-s)} A(s, \varepsilon) h_{0}(s-\Delta) d s, \quad-\sigma_{0} \leqslant t \leqslant \Delta-\sigma_{0},
$$

приходим к выводу, что

$$
\max _{t}|h(t, \varepsilon)| \leqslant M \varepsilon\left\|h_{0}\right\|, \quad M=\text { const }>0 .
$$

Для распространения оценки (2.31) на оставшийся отрезок $\left[\Delta-\sigma_{0}, T_{*}(\varepsilon)-\sigma_{0}\right]$ изменения $t$ воспользуемся методом шагов. А именно, разобьем данный промежуток 
на отрезки времени $\left[\Delta-\sigma_{0}+l \Delta, 2 \Delta-\sigma_{0}+l \Delta\right], l=0,1, \ldots, l_{0}$, и $\left[2 \Delta-\sigma_{0}+l_{0} \Delta, T_{*}(\varepsilon)-\sigma_{0}\right]$, где $l_{0}=\left\lfloor\left(T_{*}(\varepsilon)-2 \Delta\right) / \Delta\right\rfloor$ и $\lfloor\cdot\rfloor$ - целая часть. Опираясь на свойство интегральной ограниченности

$$
\int_{-\sigma_{0}}^{T_{*}(\varepsilon)-\sigma_{0}}|A(t, \varepsilon)| d t \leqslant M, \quad M=\text { const }>0
$$

имеющее место в силу (2.20)-(2.22), замечаем, что из неравенства

$$
\begin{aligned}
|h(t, \varepsilon)| \leqslant & \left|h\left(\Delta-\sigma_{0}+l \Delta, \varepsilon\right)\right| e^{-\mu\left(t-\Delta+\sigma_{0}-l \Delta\right)}+ \\
& +r \int_{\Delta-\sigma_{0}+l \Delta}^{t} e^{-\mu(t-s)}|A(s, \varepsilon)| \cdot|h(s-\Delta, \varepsilon)| d s,
\end{aligned}
$$

справедливого при $t \geqslant \Delta-\sigma_{0}+l \Delta$, и из уже полученной оценки вида (2.31) на $(l-1)$-м отрезке вытекает требуемая оценка на $l$-м отрезке изменения $t$. Лемма 2.2 доказана.

Перейдем теперь непосредственно к интересующему нас вопросу об асимптотическом вычислении мультипликаторов уравнения (2.18). С этой целью рассмотрим оператор монодромии $W(\varepsilon)$ данного уравнения, действующий в пространстве $C\left[-\Delta-\sigma_{0},-\sigma_{0}\right]$ (над полем комплексных чисел) по правилу

$$
W(\varepsilon) h_{0}=h\left(t+T_{*}(\varepsilon), \varepsilon\right), \quad-\Delta-\sigma_{0} \leqslant t \leqslant-\sigma_{0},
$$

где $h(t, \varepsilon), t \geqslant-\sigma_{0},-$ решение уравнения (2.18) с произвольной начальной функцией $h_{0}(t) \in C\left[-\Delta-\sigma_{0},-\sigma_{0}\right]$, а $T_{*}(\varepsilon)-$ период цикла $x_{*}(t, \varepsilon)$. Далее, обозначим через $\nu_{s}(\varkappa, \varepsilon), s \in \mathbb{N}$, собственные значения оператора $(2.32)$, пронумерованные в порядке убывания модулей. Справедливо следующее утверждение.

ТЕОрема 2.2. Для любого $r>0$ найдутся такие $\varepsilon_{0}=\varepsilon_{0}(r)>0, M=M(r)>0$, что при всех $0<\varepsilon \leqslant \varepsilon_{0}, \varkappa \in B(r)$ выполняется неравенство

$$
\sup _{s \geqslant 2}\left|\nu_{s}(\varkappa, \varepsilon)\right| \leqslant M \sqrt{\varepsilon}
$$

Мультипликатор $\nu_{1}(\varkappa, \varepsilon)$ допускает равномерное по параметру $\varkappa \in B(r)$ асимптотическое представление

$$
\nu_{1}(\varkappa, \varepsilon)=\left(1+\beta_{1}(\varkappa-1)\right)\left(1+\beta_{2}(\varkappa-1)\right)+O(\sqrt{\varepsilon}),
$$

¿əe

$$
\beta_{1}=\frac{1+1 / a}{1+\left(1-e^{-\mu \Delta}\right) / a}, \quad \beta_{2}=\frac{1+a}{1+a\left(1-e^{-\mu \Delta}\right)} .
$$

ДокАЗАТЕЛЬСтво. Как и при обосновании леммы 2.2, фиксируем произвольно $r>0$ и будем считать, что комплексный параметр $\varkappa$ пробегает шар $B(r)$. Введем в рассмотрение конечномерный оператор

$$
\widetilde{W}(\varepsilon) h_{0}=h_{0}\left(-\sigma_{0}\right) \tilde{h}\left(t+T_{*}(\varepsilon), \varepsilon\right), \quad-\Delta-\sigma_{0} \leqslant t \leqslant-\sigma_{0},
$$


где $\tilde{h}(t, \varepsilon)$ - решение уравнения $(2.18)$ на отрезке $-\sigma_{0} \leqslant t \leqslant T_{*}(\varepsilon)-\sigma_{0}$ с начальной функцией $\tilde{h} \equiv 1,-\Delta-\sigma_{0} \leqslant t \leqslant-\sigma_{0}$.

Остановимся на вопросе о связи между операторами (2.32) и (2.36). С этой целью рассмотрим функцию

$$
h(t, \varepsilon)-h_{0}\left(-\sigma_{0}\right) \tilde{h}(t, \varepsilon)
$$

и заметим, что при $t \in\left[-\sigma_{0}, T_{*}(\varepsilon)-\sigma_{0}\right]$ она также является решением уравнения (2.18), а при $t=-\sigma_{0}$ обращается в нуль. Тем самым мы вправе применить к функции (2.37) оценку (2.30), из которой следует, что

$$
\|W(\varepsilon)-\widetilde{W}(\varepsilon)\|_{C\left[-\Delta-\sigma_{0},-\sigma_{0}\right] \rightarrow C\left[-\Delta-\sigma_{0},-\sigma_{0}\right]} \leqslant M \varepsilon,
$$

где универсальная константа $M>0$ зависит лишь от выбора $r$.

На следующем этапе доказательства изучим спектральные свойства оператора (2.36). Нетрудно увидеть, что его спектр состоит из двух точек - собственного значения $\nu=\nu_{*}(\varkappa, \varepsilon) \stackrel{\text { def }}{=} \tilde{h}\left(T_{*}(\varepsilon)-\sigma_{0}, \varepsilon\right)$ (в общем случае простого) и собственного значения $\nu=0$ бесконечной кратности. Ниже будет показано, что для собственного значения $\nu_{*}(\varkappa, \varepsilon)$ имеет место равномерное по $\varkappa \in B(r)$ асимптотическое равенство

$$
\nu_{*}(\varkappa, \varepsilon)=\left(1+\beta_{1}(\varkappa-1)\right)\left(1+\beta_{2}(\varkappa-1)\right)+O(\sqrt{\varepsilon}),
$$

где $\beta_{1}, \beta_{2}-$ постоянные (2.35).

Для обоснования соотношения (2.39) нам необходимо знать асимптотическое поведение решения $\tilde{h}(t, \varepsilon)$. В связи с этим дополним уравнение (2.18) начальным условием $h \equiv 1,-\Delta-\sigma_{0} \leqslant t \leqslant-\sigma_{0}$, и проинтегрируем это уравнение на отрезке $-\sigma_{0} \leqslant t \leqslant T_{*}(\varepsilon)-\sigma_{0}$ методом шагов, учитывая дельтаобразное поведение коэффициента $A(t, \varepsilon)$ (см. (2.20)-(2.22)). В результате убеждаемся, что, во-первых,

$$
\max _{-\sigma_{0} \leqslant t \leqslant T_{*}(\varepsilon)-\sigma_{0}}|\tilde{h}(t, \varepsilon)| \leqslant M, \quad M=\text { const }>0,
$$

во-вторых, равномерно по $t \in \Sigma, \varkappa \in B(r)$

$$
\tilde{h}(t, \varepsilon)=h(t)+O(\sqrt{\varepsilon})
$$

где $\Sigma$ - множество из теоремы 2.1 , а через $h(t), t \geqslant-\sigma_{0}$, обозначено решение импульсной задачи Коши

$$
\begin{aligned}
\dot{h} & =-\mu h,\left.\quad h\right|_{t=-\sigma_{0}}=1, \\
h(\Delta+0) & =h(\Delta-0)-\varkappa(1+a) h(0), \\
h\left(t_{0}+\Delta+0\right) & =h\left(t_{0}+\Delta-0\right)-\varkappa(1+1 / a) h\left(t_{0}\right) .
\end{aligned}
$$

Что касается свойства (2.39), то оно очевидным образом следует из (2.41) и из равенства $h\left(T_{0}-\sigma_{0}\right)=\left(1+\beta_{1}(\varkappa-1)\right)\left(1+\beta_{2}(\varkappa-1)\right)$, которое легко проверить путем интегрирования системы (2.42) (соответствующие вполне понятные выкладки опустим). 
Вернемся к оператору $W(\varepsilon)$. Объединяя оценку (2.38) с проделанным выше асимптотическим анализом (см. (2.39), (2.40)), приходим к выводу, что все собственные значения этого оператора заведомо принадлежат шарам вида

$$
\left\{\nu \in \mathbb{C}:|\nu| \leqslant M_{1} \sqrt{\varepsilon}\right\}, \quad\left\{\nu \in \mathbb{C}:\left|\nu-\nu_{*}(\varkappa, \varepsilon)\right| \leqslant M_{2} \sqrt{\varepsilon}\right\}, \quad M_{1}, M_{2}=\text { const }>0 .
$$

Отсюда и из (2.39) требуемые соотношения (2.33), (2.34) вытекают очевидным образом. Теорема 2.2 доказана.

\section{3. ИТОГОВЫЕ РЕЗУЛЬТАТЫ}

Обратимся теперь к исходной системе (1.27) и напомним, что в силу леммы 1.1 проблема существования ее решений в виде бегущих волн сводится к отысканию периодических решений вспомогательного уравнения (2.2) (при $\varepsilon=1 / \lambda \ll 1$ ), имеющих периоды $m \Delta / k, k \in \mathbb{N}$. В связи с этим в дальнейшем периодическое решение уравнения (2.2), доставляемое теоремой 2.1, и его период будем обозначать через $x_{*}(t, \varepsilon, \Delta)$ и $T_{*}(\varepsilon, \Delta)$ соответственно, подчеркивая явно зависимость указанных функций от $\Delta$. Аналогичным образом через $T_{0}(\Delta)$ обозначим период функции (2.16), для которого в силу (2.11), (2.14) справедлива формула

$$
T_{0}(\Delta)=2 \Delta+\frac{1}{\mu} \ln \left[\left(1+\frac{1-z}{a}\right)(1+a(1-z))\right], \quad z=e^{-\mu \Delta} .
$$

Изучим сначала вопрос о существовании периодического решения с периодом $m \Delta / k$ у релейного уравнения (2.4). С этой целью обратимся к аналогичному (1.6) уравнению

$$
T_{0}(\Delta)=\frac{m \Delta}{k}
$$

для нахождения $\Delta>0$ и заметим, что в силу (3.1) оно эквивалентно уравнению

$$
\psi(z) \stackrel{\text { def }}{=} z^{2-m / k}-\left(1+\frac{1-z}{a}\right)(1+a(1-z))=0
$$

относительно $z \in(0,1)$.

ЛЕмма 3.1. При любом натуралъном $k$, принадлежащем множеству

$$
k: \frac{m}{2+a+1 / a}<k<\frac{m}{2},
$$

уравнение (3.3) допускает единственное решение $z=z_{(k)} \in(0,1)$ такое, что

$$
m\left(z_{(k)}\right)^{2}-(m-k)\left(2+a+\frac{1}{a}\right) z_{(k)}+(m-2 k)\left(2+a+\frac{1}{a}\right)>0 .
$$

В случаях $k \geqslant m / 2$ или $k \leqslant m /(2+a+1 / a)$ равенство нулю левой части (3.5) невозможно на интервале $(0,1)$. 
ДокАЗАТЕЛЬСТво. При $k \geqslant m / 2$ имеем $\psi^{\prime}(z)>0$ для всех $z \in[0,1], \psi(1)=0$, следовательно, для всех $z \in(0,1)$ выполняется неравенство $\psi(z)<0$. Аналогичным образом в случае $k \leqslant m /(2+a+1 / a)$ из очевидного неравенства $\psi^{\prime \prime}(z)>0, z \in(0,1)$, и условий $\psi(1)=0, \psi^{\prime}(1) \leqslant 0$ заключаем, что $\psi(z)>0$ для всех $z \in(0,1)$.

Перейдем теперь к интервалу значений (3.4). В этом случае функция $\psi(z)$ обладает следующими свойствами. Во-первых, справедливы соотношения

$$
\psi(1)=0, \quad \psi^{\prime}(1)>0, \quad \lim _{z \rightarrow+0} \psi(z)=+\infty,
$$

из которых автоматически вытекает существование у уравнения (3.3) хотя бы одного корня $z=z_{(k)} \in(0,1)$. Во-вторых, несложно увидеть, что уравнение

$$
\psi^{\prime}(z)=\left(2-\frac{m}{k}\right) z^{1-m / k}+2+a+\frac{1}{a}-2 z=0
$$

имеет на интервале $(0,1)$ единственный корень $z=z_{*}$, причем

$$
\psi^{\prime}(z)>0 \quad \text { при } \quad z_{*}<z \leqslant 1, \quad \psi^{\prime}(z)<0 \quad \text { при } 0<z<z_{*} .
$$

Из свойств $(3.6),(3.8)$ следует, что решение $z=z_{(k)}$ уравнения (3.3), о котором говорилось выше, единственно и

$$
\psi^{\prime}\left(z_{(k)}\right)<0 .
$$

Искомое неравенство (3.5) получается из (3.9) с учетом явной формулы (3.7) для $\psi^{\prime}(z)$ и соотношения

$$
z_{(k)}^{1-m / k}=\frac{1}{z_{(k)}}\left(1+\frac{1-z_{(k)}}{a}\right)\left(1+a\left(1-z_{(k)}\right)\right) .
$$

Лемма 3.1 доказана.

Установленная лемма гарантирует, что при любом натуральном $k$ из множества (3.4) и при $\Delta=\widehat{\Delta}_{(k)}>0$, где $\widehat{\Delta}_{(k)}=(1 / \mu) \ln \left(1 / z_{(k)}\right)$, периодическое решение (2.16) уравнения (2.4) имеет нужный период $m \widehat{\Delta}_{(k)} / k$. Обратимся теперь к периодическому решению $x_{*}(t, \varepsilon, \Delta)$ уравнения $(2.2)$ и рассмотрим соответствующее ему уравнение

$$
T_{*}(\varepsilon, \Delta)=\frac{m \Delta}{k},
$$

где номер $k$ по-прежнему принадлежит множеству (3.4). Напомним, что в силу $(2.17)$ период $T_{*}(\varepsilon, \Delta)$ допускает асимптотическое представление

$$
T_{*}(\varepsilon, \Delta)=T_{0}(\Delta)+O\left(\varepsilon \ln \frac{1}{\varepsilon}\right),
$$

причем эта асимптотика равномерна по $\Delta$ из любого компакта $K \subset(0,+\infty)$. Отсюда и из того, что корень $\Delta=\widehat{\Delta}_{(k)}$ уравнения (3.2) простой, очевидным образом заключаем, что уравнение (3.10) имеет хотя бы один корень $\Delta=\widehat{\Delta}_{(k)}(\varepsilon)$ с асимптотикой

$$
\widehat{\Delta}_{(k)}(\varepsilon)=\widehat{\Delta}_{(k)}+O\left(\varepsilon \ln \frac{1}{\varepsilon}\right) .
$$

Объединяя проделанные построения, приходим к следующему утверждению. 
Теорема 3.1. Найдется такое достаточно большое $\lambda_{0}>0$, что при всех $\lambda \geqslant \lambda_{0}$ и при любом $k$ из множества (3.4) система (1.27) допускает цзикл (бегущую волну)

$$
C_{k}: u_{j}=u_{(k)}\left(t+(j-1) \Delta_{(k)}(\lambda), \lambda\right), \quad j=1, \ldots, m,
$$

əde

$$
\Delta_{(k)}(\lambda)=\left.\widehat{\Delta}_{(k)}(\varepsilon)\right|_{\varepsilon=1 / \lambda}, \quad u_{(k)}(t, \lambda)=\left.\lambda x_{*}(t, \varepsilon, \Delta)\right|_{\varepsilon=1 / \lambda, \Delta=\Delta_{(k)}(\lambda)},
$$

$a \widehat{\Delta}_{(k)}(\varepsilon)-$ корень (3.11) уравнения (3.10).

Интересно отметить, что при $m \rightarrow \infty$ количество номеров $k$ из множества (3.4) стремится к бесконечности. Это означает, что при согласованном увеличении значений $m$ и $\lambda$ количество сосуществующих в системе (1.27) циклов (3.12) неограниченно растет. Однако, как будет показано ниже, устойчивым среди них является только цикл $C_{k}$ при условии $m=2 k+1$.

В силу леммы 1.2 проблема устойчивости цикла (3.12) с номером $k$ сводится к анализу расположения корней уравнений

$$
\left.\hat{\nu}_{s}^{k}(\varkappa, \varepsilon)\right|_{\varepsilon=1 / \lambda}=\varkappa^{m}, \quad s \in \mathbb{N},
$$

где через $\hat{\nu}_{s}(\varkappa, \varepsilon)$ обозначены мультипликаторы $\nu_{s}(\varkappa, \varepsilon)$ уравнения (2.18) при значении $\Delta=\widehat{\Delta}_{(k)}(\varepsilon)$ параметра $\Delta$. Для решения поставленной проблемы нам потребуется следующая

ЛЕмма 3.2. При каждом натуральном $k$, удовлетворяющем требованиям (3.4), и при любых значениях параметров $\beta_{1}, \beta_{2}$ таких, что

$$
\beta_{j}>1, \quad j=1,2, \quad \beta_{1}+\beta_{2}<\frac{m}{k},
$$

уравнение

$$
P(\varkappa) \stackrel{\text { def }}{=}\left(1+\beta_{1}(\varkappa-1)\right)^{k}\left(1+\beta_{2}(\varkappa-1)\right)^{k}-\varkappa^{m}=0
$$

имеет простой единичный корень, а все остальные его корни распадаются на два семейства $\Omega_{1} \subset\{\varkappa \in \mathbb{C}:|\varkappa|<1\}$ u $\Omega_{2} \subset\{\varkappa \in \mathbb{C}:|\varkappa|>1\}$. При этом множество $\Omega_{1}$ содержит $2 k$ элементов, а $\Omega_{2}$ состоит из $m-2 k-1$ корней и в случае $m=2 k+1$ оказывается пустым.

ДокАЗАТЕЛЬСтво. Из явного вида полинома $P(\varkappa)$ очевидным образом следует, что $P(1)=0$. Кроме того, в силу (3.14) имеем $P^{\prime}(1)=k\left(\beta_{1}+\beta_{2}\right)-m<0$. Что касается остальных корней уравнения (3.15), то их исследование начнем со случая

$$
\beta_{1}=1+\varepsilon_{1}, \quad \beta_{2}=1+\varepsilon_{2}, \quad 0<\varepsilon_{1}, \varepsilon_{2} \ll 1 .
$$

Нетрудно заметить, что при условиях (3.16) полином $P(\varkappa)$ имеет ровно $2 k$ корней, стремящихся к нулю при $\varepsilon_{1}, \varepsilon_{2} \rightarrow 0$. Остальные его корни (обозначим их через $\left.\varkappa_{l}\left(\varepsilon_{1}, \varepsilon_{2}\right)\right)$, отличные от единичного, при $\varepsilon_{1}=\varepsilon_{2}=0$ принимают вид $e^{i 2 \pi l /(m-2 k)}$, $l=1, \ldots, m-2 k-1$. Кроме того, несложная проверка показывает, что

$$
\left.\frac{d}{d \varepsilon_{j}}\left|\varkappa_{l}\left(\varepsilon_{1}, \varepsilon_{2}\right)\right|^{2}\right|_{\varepsilon_{1}=0, \varepsilon_{2}=0}=\frac{2 k}{m-2 k}\left(1-\cos \frac{2 \pi l}{m-2 k}\right)>0, \quad j=1,2 .
$$


Таким образом, при условиях (3.16) утверждение леммы верно.

Предположим теперь, что при некоторых значениях $\beta_{1}, \beta_{2}$, удовлетворяющих условиям (3.14), уравнение (3.15) имеет корень $\varkappa_{0}=e^{i \omega_{0}}, \omega_{0} \geqslant 0$. Тогда с необходимостью

$$
\begin{aligned}
\left|\varkappa_{0}\right|^{2 m} & =1=\left|1+\beta_{1}\left(\varkappa_{0}-1\right)\right|^{2 k}\left|1+\beta_{2}\left(\varkappa_{0}-1\right)\right|^{2 k}= \\
& =\left(1+2\left(\beta_{1}-1\right) \beta_{1}\left(1-\cos \omega_{0}\right)\right)^{k}\left(1+2\left(\beta_{2}-1\right) \beta_{2}\left(1-\cos \omega_{0}\right)\right)^{k} .
\end{aligned}
$$

Отсюда в силу неравенств $\beta_{1}, \beta_{2}>1$ очевидным образом имеем $\cos \omega_{0}=1$ и $\varkappa_{0}=1$.

Обозначим через $m_{j}$ количество корней уравнения (3.15), которые принадлежат множеству $\Omega_{j}$. Из проделанного анализа следует, что величины $m_{j}, j=1,2$, не меняются при изменении параметров $\beta_{1}, \beta_{2}$ в пределах множества, заданного условиями (3.14). Тем самым они остаются такими же, как и в случае $(3.16)$, т. е. $m_{1}=2 k$, $m_{2}=m-2 k-1$. Лемма 3.2 доказана.

Установленная лемма позволяет сформулировать свойство устойчивости циклов (3.12). Имеет место следующее утверждение.

Теорема 3.2. Цикл (3.12) с фиксированным номером $k$ экспоненциально орбитально устойчив при $m=2 k+1$ и неустойчив в противном случае.

ДокАЗАтельство. Рассмотрим систему в вариациях на цикле (3.12), имеющую вид

$$
\dot{h}_{j}=-\mu h_{j}+\hat{A}\left(t+(j-1) \widehat{\Delta}_{(k)}(\varepsilon), \varepsilon\right) h_{j-1}, \quad j=1, \ldots, m, \quad h_{0}=h_{m},
$$

где $\hat{A}(t, \varepsilon)$ - коэффициент $(2.19)$, соответствующий значению $\Delta=\widehat{\Delta}_{(k)}(\varepsilon)($ см. $(3.11))$ параметра $\Delta$, а параметр $\varepsilon=1 / \lambda$ мал. Заметим, что для оператора монодромии $\mathscr{V}(\varepsilon): \mathbb{R}^{m} \rightarrow \mathbb{R}^{m}$ системы (3.17) имеет место оценка

$$
\|\mathscr{V}(\varepsilon)\| \leqslant M, \quad M=\text { const }>0
$$

справедливость которой вытекает из свойств (2.20)-(2.22) коэффициента $\hat{A}(t, \varepsilon)$. Таким образом, при исследовании уравнений (3.13) в силу очевидного неравенства $|\nu| \leqslant\|\mathscr{V}(\varepsilon)\|$, выполняющегося для любого мультипликатора $\nu$ системы $(3.17)$, и соотношения (1.14) мы вправе ограничиться значениями параметра $\varkappa \in \mathbb{C}$ из множества $|\varkappa| \leqslant r$, где $r=(M+1)^{k / m}, M$ - константа из (3.18).

Таким образом, при условиях $\varkappa \in B(r), \Delta=\widehat{\Delta}_{(k)}(\varepsilon)$ воспользуемся теоремой 2.2 . В результате убеждаемся, что все возможные ненулевые решения $\varkappa \in \mathbb{C}$ уравнений (3.13) при $s \geqslant 2$ лежат в круге радиуса порядка $\varepsilon^{k / 2 m}$, а отвечающие им мультипликаторы $\nu=\varkappa^{m / k}$ цикла (3.12) допускают оценку вида $|\nu| \leqslant M \sqrt{\varepsilon}$ с некоторой универсальной постоянной $M>0$. Впрочем, как будет ясно из дальнейшего анализа, на самом деле мультипликаторов порядка $\sqrt{\varepsilon}$ у системы (3.17) нет, поскольку полный их набор строится уже по корням уравнения (3.13) при $s=1$.

Объединяя формулы $(2.34),(2.35),(3.11)$, приходим к выводу, что уравнение (3.13) с номером $s=1$ записывается в виде

$$
\left(1+\hat{\beta}_{1}(\varkappa-1)\right)^{k}\left(1+\hat{\beta}_{2}(\varkappa-1)\right)^{k}=\varkappa^{m}+O(\sqrt{\varepsilon}),
$$


где

$$
\hat{\beta}_{1}=\frac{1+1 / a}{1+\left(1-z_{(k)}\right) / a}, \quad \hat{\beta}_{2}=\frac{1+a}{1+a\left(1-z_{(k)}\right)},
$$

a $z_{(k)}$ - корень уравнения (3.3), доставляемый леммой 3.1. Заметим далее, что при $\varepsilon=0$ уравнение $(3.13)$ переходит в $\widehat{P}(\varkappa)=0$, где $\widehat{P}(\varkappa)$ - полином $(3.15)$ при значениях (3.20) параметров $\hat{\beta}_{1}, \hat{\beta}_{2}$. Добавим, что, так как эти значения удовлетворяют условиям (3.14) (неравенство $\hat{\beta}_{1}+\hat{\beta}_{2}<m / k$ эквивалентно оценке $(3.5)$ ), в силу леммы 3.2 интересующее нас уравнение $\widehat{P}(\varkappa)=0$ имеет простой корень $\varkappa=1$, а остальные его корни $\varkappa=\widehat{\varkappa}_{j}, j=1, \ldots, m-1$, принадлежат множествам $\Omega_{1} \subset\{\varkappa \in \mathbb{C}:|\varkappa|<1\}, \Omega_{2} \subset\{\varkappa \in \mathbb{C}:|\varkappa|>1\}$.

При $\varepsilon>0$ уравнение (3.19) также допускает решение $\varkappa=1$, поскольку при $\varkappa=1$ уравнение (2.18) заведомо имеет единичный мультипликатор (в этом случае оно представляет собой линеаризацию уравнения (2.2) на цикле, о котором идет речь в теореме 2.1). Далее, фиксируем некоторое $\delta>0$ и обозначим через $B_{\delta}(r)$ множество на комплексной плоскости переменной $\varkappa$, получающееся из $B(r)$ при выбрасывании окрестностей $\left\{\varkappa \in \mathbb{C}:\left|\varkappa-\left(\hat{\beta}_{j}-1\right) / \hat{\beta}_{j}\right|<\delta\right\}, j=1,2$. Обратим внимание на то, что при достаточно малом $\delta$ в силу очевидных неравенств $\widehat{P}\left(\left(\hat{\beta}_{j}-1\right) / \hat{\beta}_{j}\right) \neq 0$, $j=1,2$, эти окрестности заведомо не содержат корней уравнения (3.19). Тем самым в дальнейшем мы можем ограничиться рассмотрением значений $\varkappa \in B_{\delta}(r)$.

Переход от $B(r)$ к $B_{\delta}(r)$ продиктован тем обстоятельством, что в случае $\varkappa \in$ $B_{\delta}(r)$ мы имеем $\left(1+\hat{\beta}_{1}(\varkappa-1)\right)\left(1+\hat{\beta}_{2}(\varkappa-1)\right) \neq 0$. Таким образом, при $\varkappa \in B_{\delta}(r)$, $\Delta=\widehat{\Delta}_{(k)}(\varepsilon)$ мультипликатор (2.34) является простым и в силу этого аналитически зависит от $\varkappa$. Более того, справедливо аналогичное $(2.34)$ равномерное по $\varkappa \in B_{\delta}(r)$ асимптотическое равенство

$$
\frac{\partial \hat{\nu}_{1}}{\partial \varkappa}=\hat{\beta}_{1}+\hat{\beta}_{2}+2 \hat{\beta}_{1} \hat{\beta}_{2}(\varkappa-1)+O(\sqrt{\varepsilon}) .
$$

Факт аналитичности функции $\hat{\nu}_{1}(\varkappa, \varepsilon)$ по $\varkappa$ и формула $(3.21)$ позволяют утверждать, что, как и в случае $\varepsilon=0$, при $0<\varepsilon \ll 1$ количество корней уравнения (3.19) в множестве $B_{\delta}(r)$ равно $m$, причем корень $\varkappa=1$ является простым. Остальные корни при $\varepsilon \rightarrow 0$ стремятся к упомянутым выше корням $\varkappa=\widehat{\varkappa}_{j}, j=1, \ldots, m-1$, полинома $\widehat{P}(\varkappa)$. Тем самым, опираясь на леммы 1.2 и 3.2 , заключаем, что в случае $m=2 k+1$ цикл $C_{k}$ экспоненциально орбитально устойчив, а при $m \geqslant 2 k+2$ дихотомичен (с размерностью неустойчивого многообразия, равной $m-2 k$ ). Теорема 3.2 доказана.

\section{4. ЗАКЛЮЧЕНИЕ}

Обратим внимание, что теоремы $3.1,3.2$ не содержат никакой информации об аттракторах системы (1.27) при четном $m$. Для того чтобы восполнить этот пробел, при $m=2 m_{0}, m_{0} \in \mathbb{N}$, сделаем в (1.27) замены $x_{j}=u_{j} / \lambda, j=1, \ldots, 2 m_{0}$, и перейдем к пределу при $\lambda \rightarrow+\infty$. В результате с учетом равенства (2.3) получаем релейную систему

$$
\dot{x}_{j}=-\mu x_{j}+1-(a+1) F\left(x_{j-1}\right), \quad j=1, \ldots, 2 m_{0}, \quad x_{0}=x_{2 m_{0}} .
$$


Нетрудно увидеть, что данная система допускает два экспоненциально устойчивых состояния равновесия $O_{l}, l=1,2$, с координатами $x_{2 j-1}=-a / \mu, x_{2 j}=1 / \mu$, $j=1, \ldots, m_{0}$, и $x_{2 j-1}=1 / \mu, x_{2 j}=-a / \mu, j=1, \ldots, m_{0}$, соответственно. Других аттракторов у рассматриваемой системы, по всей видимости, нет. Во всяком случае, их не удалось обнаружить ни аналитическими методами, ни с помощью численного анализа, проводившегося при $m_{0}=2,3$.

Остановимся на связи наших результатов с известными результатами о существовании решений типа бегущих волн в дискретных цепочках Ферми-Паста-Улама (см., например, работы [8]-[10]). Напомним, что упомянутые цепочки имеют вид

$$
\ddot{x}_{n}=V^{\prime}\left(x_{n+1}-x_{n}\right)-V^{\prime}\left(x_{n}-x_{n-1}\right), \quad n \in \mathbb{Z},
$$

где $x_{n}=x_{n}(t) \in \mathbb{R}$ и $V(x):(0,+\infty) \rightarrow \mathbb{R}-$ гладкая функция (потенциал), а штрих обозначает дифференцирование по $x$. Интересующие нас решения типа бегущих волн задаются равенствами

$$
x_{n}(t)=n+\widehat{\varphi}(v t-n), \quad n \in \mathbb{Z}, \quad v=\text { const }>0,
$$

где функция $\widehat{\varphi}(u), u \in \mathbb{R}$ (профиль бегущей волны), при $u \rightarrow \pm \infty$ стремится к конечным пределам $\widehat{\varphi}_{ \pm}>-1$.

В статье [8] при условии $v \gg 1$ и при некоторых дополнительных ограничениях на потенциал $V(x)$ установлено существование в системе (4.2) бегущей волны (4.3) с профилем $\widehat{\varphi}(u)$, асимптотически близким к

$$
\varphi_{*}(u)= \begin{cases}-1 / 2 & \text { при } u<-1 / 2, \\ u & \text { при }-1 / 2 \leqslant u<1 / 2, \\ 1 / 2 & \text { при } u \geqslant 1 / 2 .\end{cases}
$$

Таким образом, в данной ситуации функция (4.4) играет ту же роль, что и релейное уравнение (2.4) в нашем случае, т. е. выступает в качестве достаточно простого предельного объекта.

Еще один из возможных подходов к анализу бегущих волн в цепочках (4.2) можно назвать локальным. Суть его заключается в том, что для нахождения возможных профилей $\widehat{\varphi}(u)$, удовлетворяющих дифференциально-разностному уравнению

$$
v^{2} \widehat{\varphi}^{\prime \prime}=V^{\prime}(1+\widehat{\varphi}(u-1)-\widehat{\varphi}(u))-V^{\prime}(1+\widehat{\varphi}(u)-\widehat{\varphi}(u+1)),
$$

применяется метод центральных многообразий. Следует заметить, что, хотя начальная задача Коши для уравнений вида (4.5) некорректна, теорема о существовании гладкого центрального многообразия в окрестности нулевого положения равновесия для таких уравнений справедлива (см., например, работу [11]). Тем самым проблема отыскания малых решений $\widehat{\varphi}(u), u \in \mathbb{R}$, сводится к анализу конечномерной системы обыкновенных дифференциальных уравнений, являющейся нормальной формой исходного дифференциально-разностного уравнения на упомянутом многообразии. Описанный подход, реализованный в статьях [9], [10] и в ряде других работ, вполне может быть применен и к цепочкам (1.1) однонаправленно связанных уравнений. 
В заключение отметим, что предложенные в настоящей работе методы исследования периодических решений типа бегущих волн распространяются и на цепочки уравнений с запаздыванием вида

$$
\dot{x}_{j}=f\left(x_{j}, x_{j}\left(t-h_{1}\right), x_{j-1}, x_{j-1}\left(t-h_{2}\right)\right), \quad j=1, \ldots, m, \quad x_{0}=x_{m},
$$

где $m \geqslant 2, x_{j}=x_{j}(t) \in \mathbb{R}^{n}, h_{1}, h_{2}>0$, а вектор-функция $f(x, y, z, v)$ со значениями в $\mathbb{R}^{n}$ бесконечно дифференцируема по $(x, y, z, v) \in \mathbb{R}^{4}$. Примером цепочки (4.6) служит математическая модель кольцевой нейронной сети Хопфилда с запаздыванием, представляющая собой систему

$$
\begin{aligned}
& \dot{u}_{j}=-\mu u_{j}+\lambda\left[1-(a+1) f\left(u_{j}(t-1)\right)-b g\left(u_{j-1}\right)\right], \quad j=1, \ldots, m, \\
& u_{0}=u_{m} .
\end{aligned}
$$

Здесь параметры $\mu, a, \lambda$ и функция $f(u)$ те же, что и в (1.27), $b=$ const $>0$, а для функции $g(u) \in C^{\infty}(\mathbb{R})$ справедливы аналогичные (1.28) асимптотические представления

$$
g(u)=\sum_{l=1}^{\infty} \frac{d_{l}^{-}}{u^{l}} \quad \text { при } \quad u \rightarrow-\infty, \quad g(u)=1+\sum_{l=1}^{\infty} \frac{d_{l}^{+}}{u^{l}} \quad \text { при } \quad u \rightarrow+\infty,
$$

сохраняющие силу при дифференцировании по $u$ любое число раз.

Другой пример - математическая модель кольцевой нейронной сети с однонаправленными химическими связями, имеющая вид

$$
\dot{u}_{j}=\left(\lambda f\left(u_{j}(t-1)\right)+b g\left(u_{j-1}\right) \ln \frac{u_{*}}{u_{j}}\right) u_{j}, \quad j=1, \ldots, m, \quad u_{0}=u_{m} .
$$

Здесь $\lambda \gg 1, b=$ const $>0, u_{*}=e^{c \lambda}, c=$ const $\in \mathbb{R}$, а функции $f(u)$ и $g(u) \in C^{2}\left(\mathbb{R}_{+}\right)$, $\mathbb{R}_{+}=\{u \in \mathbb{R}: u \geqslant 0\}$, удовлетворяют следующим условиям: $f(0)=1$ и $g(u)>0$ при всех $u>0, g(0)=0$,

$$
\begin{aligned}
& f(u)+a=O(1 / u), \quad u f^{\prime}(u)=O(1 / u), \quad u^{2} f^{\prime \prime}(u)=O(1 / u), \\
& g(u)-1=O(1 / u), \quad u g^{\prime}(u)=O(1 / u), \quad u^{2} g^{\prime \prime}(u)=O(1 / u), \quad u \rightarrow+\infty,
\end{aligned}
$$

где $a=$ const $>0$.

Подробному рассмотрению проблем существования и устойчивости периодических решений типа бегущих волн в системах (4.7), (4.8) будут посвящены отдельные статьи. Здесь же отметим, что, в отличие от случая (1.27), в этих цепочках при подходящем выборе параметров может сосуществовать любое наперед заданное конечное число устойчивых бегущих волн, т. е. реализуется известное явление буферности.

Благодарности. Выражаем искреннюю благодарность рецензенту за ряд полезных замечаний, позволивших улучшить текст статьи. Работа выполнена при финансовой поддержке РФФИ (гранты № 11-01-00384_а и 12-01-0015_а). 


\section{Список литературы}

[1] С. Д. Глызин, А. Ю. Колесов, Н. Х. Розов, Ж. вычисл. матем. и матем. физ., 46:10 (2006), 1809-1821.

[2] Дж. К. Спротт, Элегантный хаос: алгебраччески простые хаотические потоки, ИКИ, Ижевск, 2012.

[3] J. J. Hopfield, Proc. Nat. Acad. Sci. USA, 81:10 (1984), 3088-3092.

[4] С. Хайкин, Нейронные сети: полный курс, Издательский дом "Вильямс", М., СПб., Киев, 2006.

[5] А. Ю. Колесов, Е. Ф. Мищенко, Н. Х. Розов, Тр. МИАН, 216 (1997), 126-153.

[6] А. Ю. Колесов, Е. Ф. Мищенко, Н. Х. Розов, Ж. вычисл. матем. и матем. физ., 50:12 (2010), 2099-2112.

[7] С. Д. Глызин, А. Ю. Колесов, Н. Х. Розов, Дифберени. уравнения, 47:7 (2011), 919-932.

[8] D. Treschev, Discrete Contin. Dyn. Syst., 11:4 (2004), 867-880.

[9] G. Iooss, Nonlinearity, 13:3 (2000), 849-866.

[10] G. Iooss, K. Kirchgässner, Commun. Math. Phys., 211:2 (2000), 439-464.

[11] Th. Gallay, Commun. Math. Phys., 152:2 (1993), 249-268.

Поступила в редакцию 14.11.2012, после доработки 23.11.2012 